BNL-68295

Submitted to the Proceedings of China International Battery Fair 2001 (CIBF2001), June $8^{\text {th }}$ to June $12^{\text {th }}$, 2001, Beijing, China.

\title{
Synthesis of a New Family of Fluorinated Boronate Compounds as Anion Receptors and Studies of Their Use as Additives in Lithium Battery Electrolytes
}

\author{
J. McBreen, H. S. Lee, and X. Q. Yang \\ Brookhaven National Laboratory, Upton, NY 11973
}

\section{Introduction}

Numerous studies have been done on developing new electrolytes for lithium batteries with high ionic conductivity, and good chemical and electrochemical stability. In addition to the research on new salts and solvents, the use of cation receptors to reduce ion pairing in non-aqueous electrolytes has been considered as an approach to improve the properties of electrolytes ${ }^{1}$. Although both cation and anion receptors enhance the dissociation of ion pairs and increase the conductivity of electrolytes, the use of anion receptors is more attractive for a lithium battery electrolyte because anion receptors increase the lithium transference number in the electrolyte. However, most available neutral anion receptors complex with anions through hydrogen binding and cannot be used in lithium batteries ${ }^{2}$. Recently, we have reported on synthesis of a series of new neutral boron compounds as anion receptors based on the idea that electron-deficient boron would complex the anion of the ion pair. The anion complexation effect of these boron compounds was further enhanced by attaching electronwithdrawing groups $\mathrm{s}^{3,4}$. Here we report synthesis of another new family of boronate compounds. The effect of these new compounds on conductivity of lithium salts in non-aqueous solution was studied. The molecular weights of these new boronate compounds are lower than our previously reported boron compounds. Therefore, their effects on conductivity enhancement are superior. They also display high electrochemical stability up to $5 \mathrm{~V}$.

\section{Experimental}

Nine fluorinated 1,3,2-benzodioxaborole and 1,3,2-Dioxaborolane compounds were synthesized in our laboratory. The chemical structure of these compounds was shown in Figure 1. The synthesis procedure of these compounds can be found in our early publication ${ }^{5}$.

Electrolyte conductivity measurements were made on a Hewlett-Packard 4129A Impedance Analyzer using a cell with Pt electrodes. Electrochemical stability measurements were performed using a Solatron SI 1287 Electrochemical Interface in the potential dynamic mode.

\section{Results and Discussion}

The ionic conductivity of electrolytes based on the lithium salts, $\mathrm{CF}_{3} \mathrm{CO}_{2} \mathrm{Li}$ and $\mathrm{C}_{2} \mathrm{~F}_{5} \mathrm{CO}_{2} \mathrm{Li}$, dissolved in DME are very low, with respective conductivity of only $3.3 \times 10^{-5}$ and $2.1 \times 10^{-5} \mathrm{~S} / \mathrm{cm}$ at concentrations of 0.2 $\mathrm{M}$. $\mathrm{LiF}$ is insoluble in this solvent. With addition of stoichiometric amounts of compound $\mathbf{1}$ as an additive, the conductivity of $0.2 \mathrm{M}$ solutions of $\mathrm{CF}_{3} \mathrm{CO}_{2} \mathrm{Li}$ and $\mathrm{C}_{2} \mathrm{~F}_{5} \mathrm{CO}_{2} \mathrm{Li}$ salts in DME were greatly increased to $1.24 \times 10^{-}$ 
3 and $1.1 \times 10^{-3} \mathrm{~S} / \mathrm{cm}$ respectively, as shown in Table I. The conductivity enhancement effect of these compounds increases with increasing number of the electron-withdrawing groups. When fully fluorinated compound 5 was used as an additive, even LiF was dissolved in DME at concentrations as high as 1.2 M, and the conductivity of LiF solution in DME reached as high as $9.54 \times 10^{-3} \mathrm{~S} / \mathrm{cm}$ at $0.8 \mathrm{M}$, as shown in Table II.

The electrochemical stability studies were carried out using a Solatron SI 1287 Electrochemical Interface in potential dynamic mode at a scan rate of $20 \mathrm{mV} / \mathrm{s}$. The resulting curve is plotted in Figure 2. The electrolyte made of compound (9), LiF salt and EC/DMC solvent has excellent electrochemical stability at voltage vs $\mathrm{Li}$ as high as $5 \mathrm{~V}$.

\section{Acknowledgement}

This work was supported by the U. S. Department of Energy Division of Materials Science of the Office Basic Energy Sciences, and the Office of Energy Research, Laboratory Technology Research Program, under Contract No. DE-AC02-98CH10886.

\section{References}

1. M. C. Lonergan, M. A. Ratner, D. Shriver, J. Am. Chem. Soc., 117, 2344 (1995).

2. F.P. Schmidtchen and M. Berger, Chemical Reviews, 97, 1609 (1997).

3. H. S. Lee, X. Q. Yang, C. L. Xiang, J. McBreen, L. S. Choi, J. Electrochem. Soc., 145, 2813 (1998).

4. X. Sun, H. S. Lee, S. Lee, X. Q. Yang and J. McBreen, Electrochem. Solid State. Lett., 1, 239 (1998).

5. H. S. Lee, X. Q. Yang, X. Sun, and J. McBreen, Journal of Power Source, accepted

\section{Figure 1. Chemical Structures of Boronate Based Anion Receptors}

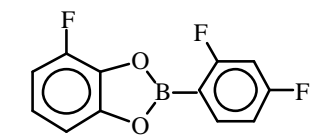

$1\left(\mathrm{C}_{6} \mathrm{H}_{3} \mathrm{~F}\right) \mathrm{O}_{2} \mathrm{~B}\left(\mathrm{C}_{6} \mathrm{H}_{3} \mathrm{~F}_{2}\right)$

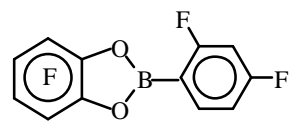

$4\left(\mathrm{C}_{6} \mathrm{~F}_{4}\right) \mathrm{O}_{2} \mathrm{~B}\left(\mathrm{C}_{6} \mathrm{H}_{3} \mathrm{~F}_{2}\right)$

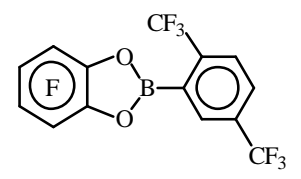

$7\left(\mathrm{C}_{6} \mathrm{~F}_{4}\right) \mathrm{O}_{2} \mathrm{~B}\left(\mathrm{C}_{8} \mathrm{H}_{3} \mathrm{~F}_{6}\right)$

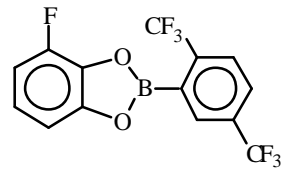

$2\left(\mathrm{C}_{6} \mathrm{H}_{3} \mathrm{~F}\right) \mathrm{O}_{2} \mathrm{~B}\left(\mathrm{C}_{8} \mathrm{H}_{3} \mathrm{~F}_{6}\right)$

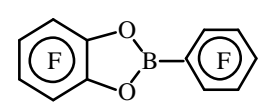

$5\left(\mathrm{C}_{6} \mathrm{~F}_{4}\right) \mathrm{O}_{2} \mathrm{~B}\left(\mathrm{C}_{6} \mathrm{~F}_{5}\right)$

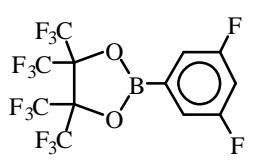

$8\left(\mathrm{C}_{6} \mathrm{~F}_{12}\right) \mathrm{O}_{2} \mathrm{~B}\left(\mathrm{C}_{6} \mathrm{H}_{3} \mathrm{~F}_{2}\right)$

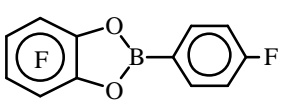

$3\left(\mathrm{C}_{6} \mathrm{~F}_{4}\right) \mathrm{O}_{2} \mathrm{~B}\left(\mathrm{C}_{6} \mathrm{H}_{4} \mathrm{~F}\right)$

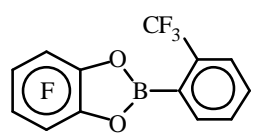

$6\left(\mathrm{C}_{6} \mathrm{~F}_{4}\right) \mathrm{O}_{2} \mathrm{~B}\left(\mathrm{C}_{7} \mathrm{H}_{4} \mathrm{~F}_{3}\right)$

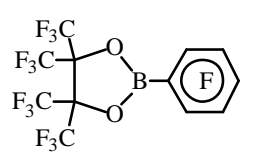

$9\left(\mathrm{C}_{6} \mathrm{~F}_{12}\right) \mathrm{O}_{2} \mathrm{~B}\left(\mathrm{C}_{6} \mathrm{~F}_{5}\right)$ 
Figure 2. Electrochemical stability of boronate-based anion receptors

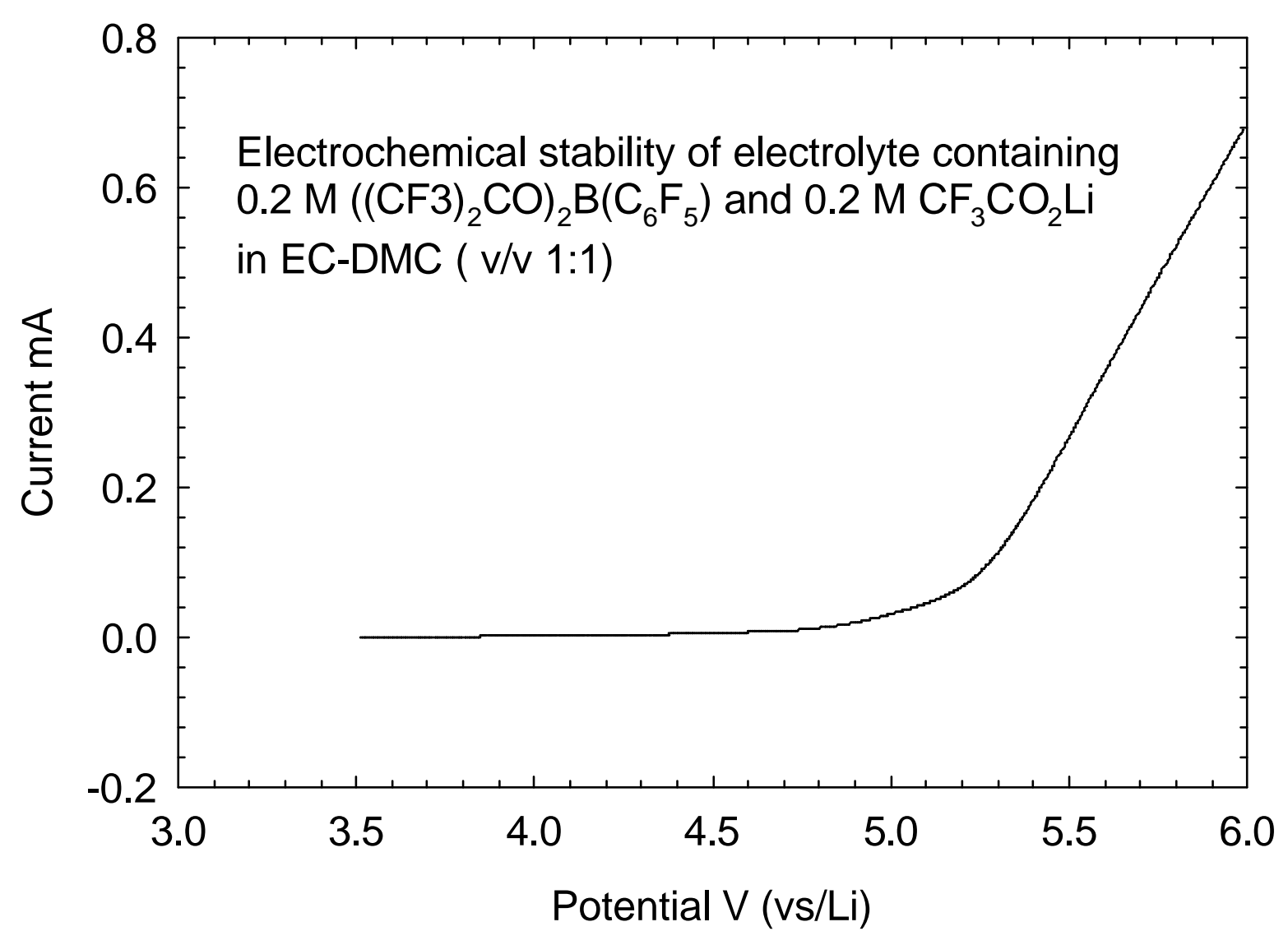


Table I. Ionic Conductivity of Compound (1) + Lithium Salt in DME solutions

\begin{tabular}{|c|c|c|c|c|}
\hline $\begin{array}{l}\text { Concentration } \\
\text { of } \\
\text { Compound (1) }\end{array}$ & $\begin{array}{c}\text { Concentration } \\
\text { of } \\
\text { Lithium Salt }\end{array}$ & $\begin{array}{l}\text { Conductivity } \\
\mathrm{CF}_{3} \mathrm{COOLi} \\
(\mathrm{S} / \mathrm{cm})\end{array}$ & $\begin{array}{c}\text { Conductivity } \\
\mathrm{C}_{2} \mathrm{~F}_{5} \mathrm{COOLi} \\
(\mathrm{S} / \mathrm{cm})\end{array}$ & $\begin{array}{c}\text { Conductivity } \\
\text { LiF } \\
\text { (S/cm) }\end{array}$ \\
\hline 0 & $0.2 \mathrm{M}$ & $3.3 \times 10^{-5}$ & $2.1 \times 10^{-5}$ & $*$ \\
\hline $0.2 \mathrm{M}$ & $0.2 \mathrm{M}$ & $1.24 \times 10^{-3}$ & $1.1 \times 10^{-3}$ & $*$ \\
\hline $0.4 \mathrm{M}$ & $0.4 \mathrm{M}$ & $2.83 \times 10^{-3}$ & $2.73 \times 10^{-3}$ & * \\
\hline $0.6 \mathrm{M}$ & $0.6 \mathrm{M}$ & $3.83 \times 10^{-3}$ & $3.69 \times 10^{-3}$ & * \\
\hline $0.8 \mathrm{M}$ & $0.8 \mathrm{M}$ & $4.20 \times 10^{-3}$ & $4.07 \times 10^{-3}$ & * \\
\hline $1.0 \mathrm{M}$ & $1.0 \mathrm{M}$ & $3.96 \times 10^{-3}$ & $3.86 \times 10^{-3}$ & * \\
\hline $1.2 \mathrm{M}$ & $1.2 \mathrm{M}$ & $3.40 \times 10^{-3}$ & $3.35 \times 10^{-3}$ & $*$ \\
\hline
\end{tabular}

* The solubility of LiF was very low, therefore, the conductivity was not measured.

Table II. Ionic Conductivity of Compound (5) + Lithium Salt in DME solutions

\begin{tabular}{|c|c|c|c|c|}
\hline $\begin{array}{l}\text { Concentration } \\
\text { of } \\
\text { Compound (5) }\end{array}$ & $\begin{array}{c}\text { Concentration } \\
\text { of } \\
\text { Lithium Salt }\end{array}$ & $\begin{array}{c}\text { Conductivity } \\
\mathrm{CF}_{3} \mathrm{COOLi} \\
(\mathrm{S} / \mathrm{cm})\end{array}$ & $\begin{array}{c}\text { Conductivity } \\
\mathrm{C}_{2} \mathrm{~F}_{5} \mathrm{COOLi} \\
(\mathrm{S} / \mathrm{cm})\end{array}$ & $\begin{array}{c}\text { Conductivity } \\
\text { LiF } \\
\text { (S/cm) }\end{array}$ \\
\hline 0 & $0.2 \mathrm{M}$ & $3.3 \times 10^{-5}$ & $2.1 \times 10^{-5}$ & $*$ \\
\hline $0.2 \mathrm{M}$ & $0.2 \mathrm{M}$ & $4.11 \times 10^{-3}$ & $4.52 \times 10^{-3}$ & $3.58 \times 10^{-3}$ \\
\hline $0.4 \mathrm{M}$ & $0.4 \mathrm{M}$ & $7.51 \times 10^{-3}$ & $7.67 \times 10^{-3}$ & $6.85 \times 10^{-3}$ \\
\hline $0.6 \mathrm{M}$ & $0.6 \mathrm{M}$ & $9.00 \times 10^{-3}$ & $8.92 \times 10^{-3}$ & $8.98 \times 10^{-3}$ \\
\hline $0.8 \mathrm{M}$ & $0.8 \mathrm{M}$ & $8.24 \times 10^{-3}$ & $8.10 \times 10^{-3}$ & $9.54 \times 10^{-3}$ \\
\hline $1.0 \mathrm{M}$ & $1.0 \mathrm{M}$ & $7.76 \times 10^{-3}$ & $6.49 \times 10^{-3}$ & $9.00 \times 10^{-3}$ \\
\hline $1.2 \mathrm{M}$ & $1.2 \mathrm{M}$ & $6.62 \times 10^{-3}$ & $4.67 \times 10^{-3}$ & $7.68 \times 10^{-3}$ \\
\hline
\end{tabular}

* The solubility of LiF was very low , therefore, the conductivity was not measured. 